\title{
Rate of uncontrolled blood pressure and its associated factors in patients with predialysis chronic kidney disease in Bali, Indonesia
}

\author{
Ni Wayan Kesari Dharmapatni \\ Faculty of Nursing, Mahidol University, Bangkok, Thailand, and \\ Aurawamon Sriyuktasuth and Kanaungnit Pongthavornkamol \\ Department of Medical Nursing, Faculty of Nursing, Mahidol University, \\ Bangkok, Thailand
}

\begin{abstract}
Purpose - Hypertension is a key determinant for the development and progression of chronic kidney disease (CKD). The purpose of this study is to assess the rate of uncontrolled blood pressure (BP) and identify its associated factors in patients with predialysis CKD in Bali, Indonesia.

Design/methodology/approach - A cross-sectional study was conducted among 165 patients who attended the nephrology clinic in a central public hospital in Bali. Data were obtained by measuring BP at threshold 130/80 mmHg, as well as collected through standardized questionnaires. Univariate analysis was done using Chi-square test, and multivariate analyses were carried out using multiple logistic regression.

Findings - A total of 165 patients (111 males and 54 females) with predialysis CKD participated in this study. About $64 \%$ of the participants had uncontrolled BP. In multiple logistic regression, all selected variables significantly explained $63.2 \%$ of the variance in uncontrolled BP. However, low physical activity (odds ratio $[\mathrm{OR}]=24.287,95 \%$ confidence interval $[\mathrm{C}]$ ]: $3.114-189.445)$, unhealthy dietary pattern $(\mathrm{OR}=10.153,95 \% \mathrm{CI}$ : $2.770-37.210)$, as well as perceived moderate stress $(\mathrm{OR}=4.365,95 \% \mathrm{CI}$ : 1.024-18.609) and high stress $(\mathrm{OR}=10.978,95 \%$ CI: 2.602-46.312) were significantly associated with uncontrolled BP.

Research limitations/implications - The study findings provide evidence for health care providers to improve $\mathrm{BP}$ control among patients with predialysis CKD.

Originality/value - Controlling BP among patients with predialysis CKD was poor. Lifestyle modification and stress management are keys to improving BP control.
\end{abstract}

Keywords Blood pressure, Predialysis, Chronic kidney disease, Indonesia

Paper type Research paper

\section{Introduction}

Chronic kidney disease (CKD) is a leading cause of morbidity and mortality globally. It is estimated that 497.5 million people in the world have $\mathrm{CKD}$ and that this number is expected to increase over time [1]. Approximately $80 \%$ of the disease burden happens in low- or middleincome countries and since 1990 , the CKD burden contributed to an $82 \%$ increase in mortality [2].

(C) Ni Wayan Kesari Dharmapatni, Aurawamon Sriyuktasuth and Kanaungnit Pongthavornkamol. Published in Journal of Health Research. Published by Emerald Publishing Limited. This article is published under the Creative Commons Attribution (CC BY 4.0) license. Anyone may reproduce, distribute, translate and create derivative works of this article (for both commercial and non-commercial purposes), subject to full attribution to the original publication and authors. The full terms of this license may be seen at http://creativecommons.org/licences/by/4.0/legalcode

This study was funded by the Indonesia Endowment Fund for Education (LPDP scholarship). The authors acknowledge Prof. Dr Alicia Matthews for constructive and valuable comments during the development of the manuscript. The authors express their thanks to the Institute of Technology and Health Bali for its support during data collection.

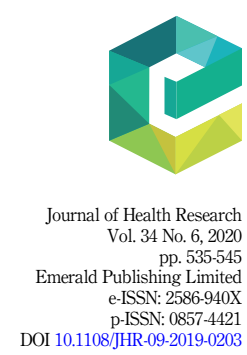

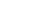


JHR 34,6

Currently, CKD is a major health, social and economic problem in Indonesia. According to Riskesdas (Basic Health Research) [3], CKD was one of the top ten noncommunicable diseases in Indonesia. Moreover, evidence indicates that over $80 \%$ of patients with CKD have hypertension $[4,5]$, and high blood pressure (BP) is a key determinant for the development and progression of CKD [6, 7]. Guidelines of the 2017 American College of Cardiology/ American Heart Association (AHA) recommends a BP goal of $<130 / 80 \mathrm{mmHg}$ for patients with CKD [8]. However, most patients have difficulty reaching this target BP level. Prior research has repeatedly found a range of $50-75 \%$ for uncontrolled BP among patients [9-11]. The evidence suggests that uncontrolled $\mathrm{BP}$ was a major risk factor for cardiovascular morbidity and mortality in the CKD population, in addition to the development of end-stage renal disease (ESRD) [1]. The prevalence of ESRD has been increasing around the world, and more than 1.9 million patients with ESRD live on dialysis [1]. Thus, uncontrolled BP should be addressed earlier in predialysis CKD.

Based on findings from review literature, BP is multifactorial, as many factors are associated with uncontrolled BP in the CKD population. The model for Hypertension Development and Assessment (HDA) was developed to understand factors contributing to the development of hypertension [12]. This model has four tiers of domains with lifestyle identified as the strongest factor, followed by response to the environment, physiology and genetic factors.

As a dynamic factor, lifestyle can be modified by healthcare providers. Lifestyle factors include physical activity [13] and dietary patterns [14], and these have been found to be important for controlling BP in the hypertensive population. Similarly, response to the environment, including perceived stress, has been found to be associated with uncontrolled BP [15]. One study explained that increased BP occurs as the reaction of perceived stress from the environment [12]. However, the results relating to the physiology and genetic factors on uncontrolled $\mathrm{BP}$ in the $\mathrm{CKD}$ population are somewhat mixed. In particular, physiological factors, such as age, stage of CKD, diabetes mellitus and cardiovascular diseases (CVDs) have been found to be inconsistent in prior studies. Older age [16], a higher stage of CKD [17], having diabetes [9] and CVD [17] have been identified as factors that are associated with uncontrolled BP. On the other hand, some studies found no association between these factors with uncontrolled BP $[6,18]$. Likewise, studies of gender as a genetic determinant had mixed results. One study found uncontrolled BP in males [17], while another study found uncontrolled BP in females [16], and some studies found that gender did not affect BP levels [9, 18]. In addition, some studies identified a patient's background characteristics such as marital status [19], education level [20] and employment [21] as associated factors of uncontrolled BP.

Although numerous studies about BP control have been conducted in patients with CKD, many of them focused on the dialysis stage. Similar studies conducted in predialysis CKD and hypertensive patients in different contexts had inconsistent findings. To date, in Indonesia, little is known about the rate of uncontrolled BP and its associated factors in patients with predialysis CKD. Therefore, additional research is needed to better understand the rate and associated factors for uncontrolled BP amongst this sector of the population.

Using the HDA model as a conceptual framework, this study aimed to assess the rate of uncontrolled BP and identify its associated factors in patients with predialysis CKD. The findings may help to provide evidence for healthcare providers to plan interventions to improve BP control in patients with predialysis CKD in Bali, Indonesia.

\section{Methods}

Study design and participants

This cross-sectional study was conducted amongst 165 conveniently selected adults at the Nephrology Clinic in the outpatient department of a central public hospital in Bali, Indonesia between January and March 2019. 
Participants included both male and female patients who were 18 years or older, diagnosed with CKD for at least three months and who had not been undergoing dialysis. CKD was defined as a glomerular filtration rate less than $60 \mathrm{ml} / \mathrm{min} / 1.73 \mathrm{~m}^{2}$ [22]. The estimated glomerular filtration rate (eGFR) was calculated from serum creatinine using the Chronic Kidney Disease Epidemiology Collaboration (CKD-EPI) equation.

Exclusion criteria in this study included having a diagnosis showing psychiatric illness, having a severe disease/condition, taking medication that influences BP (i.e. cold medicines including pseudoephedrine and phenylephrine, analgesics including NSAIDs, immunosuppressants including cyclosporine and tacrolimus, antacids and oral contraceptives), changing a antihypertensive regimen within the past three months or being elderly and not passing the screening for cognitive impairment based on the Mini-Mental State Examination (MMSE).

The sample size was calculated using GPower software based on the predictive power of variables in the study. The maximum sample size calculation was adapted from a previous study indicating the predictive power of age on uncontrolled BP with odds ratio (OR) 3.823 [4]. Other parameters included $\alpha=0.05$, power $=0.80$, probability $(Y=1 ; X=1) H 1=0.234$ and probability $(Y=1 ; X=1) H 0=0.074$ calculated according to the result of the previous study and binomial distribution. With that specification, the prescribed sample size for this study was 165 participants.

\section{Ethical consideration}

The study protocol was approved by the Institutional Review Board of the Faculty of Nursing, Mahidol University,Thailand (CoA No. IRB-NS2018/464.0911) and the Research Ethics Committee in Bali, Indonesia.

\section{Measurements}

A calibrated automated sphygmomanometer with an appropriate cuff size was used to measure BP following the AHA guidelines for BP measurement [23]. Every participant was advised to refrain from alcohol, coffee or tea intake, cigarette smoking and exercise for at least 30 min prior to BP measurement. BP was obtained in a sitting position after at least $5-10 \mathrm{~min}$ of quiet rest with legs uncrossed and the back and arm supported. BP was measured two times at 2 min intervals. An additional measurement was conducted when the two readings differed by more than $10 \mathrm{mmHg}$. All readings were averaged, and a systolic BP reading was used as the primary consideration. "Uncontrolled BP" was defined as systolic BP $\geq 130 \mathrm{mmHg}$ and/or diastolic $\mathrm{BP} \geq 80 \mathrm{mmHg}$ and was used in data analysis as the dependent variable [8].

Demographic (age, gender, marital status, educational level, employment status) and clinical (stage of CKD, diabetes mellitus, CVD) data were collected.

Perceived stress levels were assessed by the Perceived Stress Scale (PSS-10) [24] which was translated into Indonesian by the researchers. This scale is valid [25] and reliable (Cronbach's alpha 0.89) [15]. In the present study, Cronbach's alpha for the PSS was 0.87 . The PSS consisted of ten items, and the response used a 5-level Likert scale. Response options ranged from "never" (0) to "very often" (4). The score was transformed on a 0 - to 40-point scale and divided into three categories: low, moderate and high perceived stress.

Physical activity levels were assessed by the Indonesian version of the International Physical Activity Questionnaire (IPAQ) [26]. Cronbach's alpha was 0.70 in a prior study [27] and 0.72 in the current study. The IPAQ consisted of seven items, and the level of physical activity was calculated based on metabolic equivalent energy expenditure. The total score was categorized according to the official IPAQ scoring protocol as low, moderate and high physical activity [28].
Factors of uncontrolled blood pressure 
JHR 34,6

Dietary patterns were assessed by the Food Frequency Questionnaire (FFQ) [29]. The FFQ was translated into Indonesian by the researchers and modified to suit the Indonesian culture. The scale was valid [30] and the content validity index (CVI) of the modified Indonesian FFQ version was 1.0 in the current study. Cronbach's alpha was 0.83 amongst a sample of Chinese [31] and 0.78 in the present study. The modified FFQ consisted of 17 items and was rated on a 5 -level Likert scale. Response options ranged from ">6 times/week" (0) to "never" (4). The total score had a possible range of $0-68$ and could be summarized as a "healthy" and "unhealthy" dietary pattern.

\section{Participant enrollment and data collection}

A face-to-face meeting was conducted when participants visited the hospital for follow-up. The staff nurses at the study clinic identified potential participants who met the inclusion criteria. The study protocol, objectives, risks, benefits, privacy and confidentiality were explained to each potential participant by the researcher. All participants were voluntary and could withdraw their participation from the study at any time during data collection with no effect on their treatment or hospital services. The potential participants who provided written informed consent were enrolled on to the study. During data collection, all participants had their BP measured. Then, participants were asked to answer the questionnaires while waiting to visit the nephrologist. Clinical data were obtained from a patient's medical record with permission. Data were collected only once, and participants took approximately 30-40 min to complete the questionnaire. All data were collected by the principal investigator with no missing data in the current study.

\section{Data analysis}

Data analysis was performed using Statistical Package for the Social Sciences. Characteristics were summarized using means for continuous variables and counts and percentages for categorical variables. In univariate analysis, characteristics of patients were compared between controlled and uncontrolled groups by the Chi-square test. Next, the potential variables associated with uncontrolled blood pressure in univariate analysis were entered into the multiple logistic regression analysis using the enter method. Assumptions were tested for each statistical test. All reported $\mathrm{p}$ values were 2-sided and considered statistically significant at $\alpha=0.05$.

\section{Results \\ Participant demographics}

A total of 165 patients with predialysis CKD were enrolled and successfully completed the study questionnaire. Half were aged between 41 and 60 years $(50.3 \%)$ with a mean age of $55.8 \pm 12.8$ years. The majority of participants were male $(67.3 \%)$, married $(92.1 \%)$ and were educated to at least high school level $(66.0 \%)$. Also, $53.3 \%$ of the sample were unemployed (Table I).

\section{Clinical characteristics}

Many of the participants were in CKD stage $3(37.6 \%)$ and stage $5(37.0 \%)$ with a mean eGFR of $24.60 \pm 17.15$. Almost half the sample had diabetes mellitus (41.8\%) and CVD (46.7\%). Each participant had received at least one type of antihypertensive medication. Of the total sample, $64.8 \%$ had uncontrolled BP, even though they were prescribed with angiotensin-converting enzyme inhibitor, and/or angiotensin receptor blockers, and/or calcium channel blockers, and/or diuretic agents for their antihypertensive regimen. 


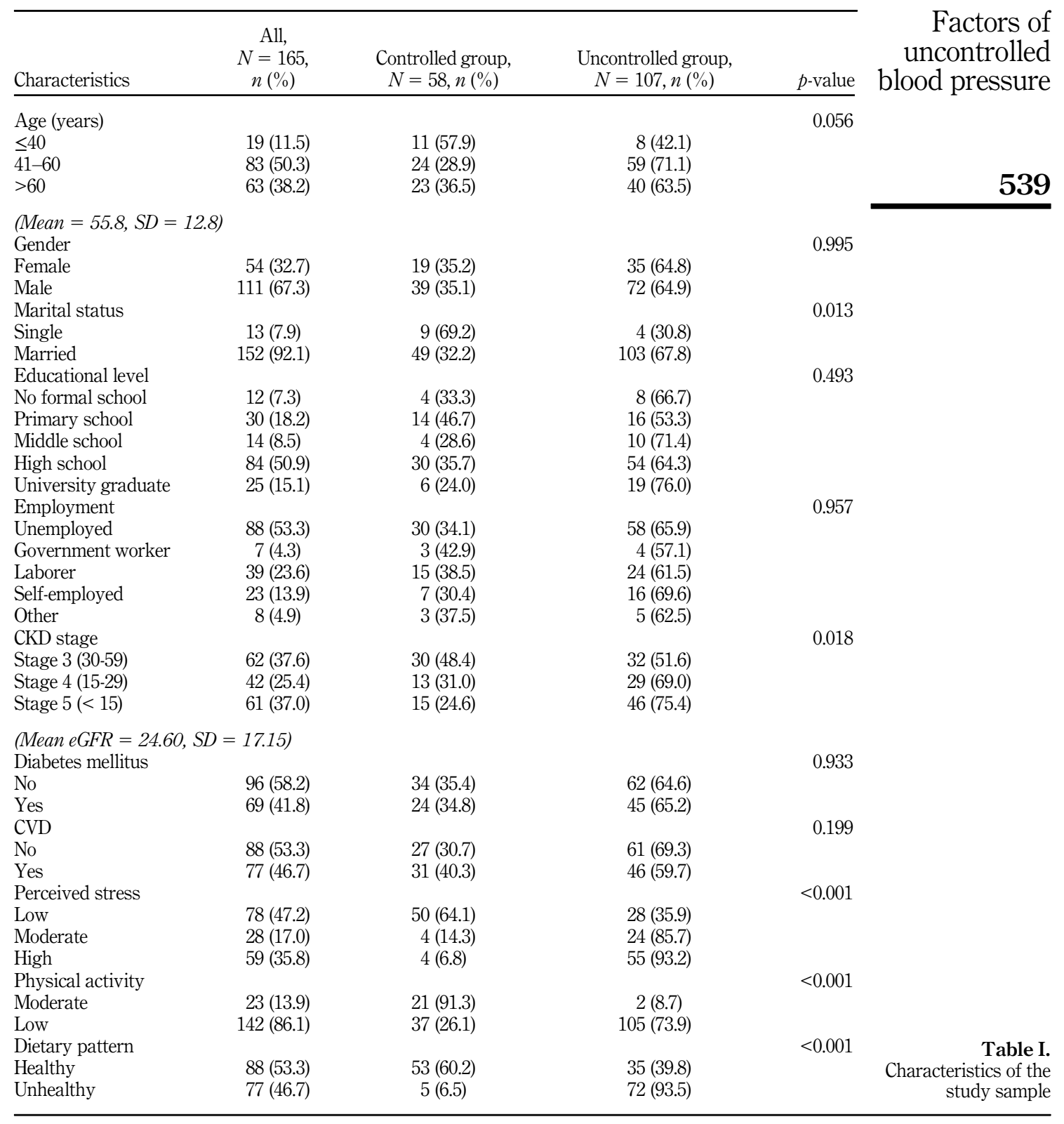

\section{Predictors of uncontrolled blood pressure}

Univariate analysis. Results from the Chi-square analysis showed that marital status $(\phi=0.013)$, stage of CKD $(p=0.018)$, perceived stress $(p<0.001)$, physical activity $(\phi<0.001)$ and dietary pattern $(\phi<0.001)$ were significantly associated with uncontrolled BP. In addition, age was associated with uncontrolled BP with a $p$-value of 0.056 (Table I). Therefore, 
JHR 34,6

540 these six potential variables associated with uncontrolled BP were entered in the multiple logistic regression analysis.

Multivariate analysis. The result of the logistic regression analysis of the six selected variables could explain $63.2 \%$ of the variance in uncontrolled BP. Perceived moderate $(\mathrm{OR}=4.365,95 \% \mathrm{CI}: 1.024-18.609)$ and high stress $(\mathrm{OR}=10.978,95 \% \mathrm{CI}: 2.602-46.312)$, low physical activity $(\mathrm{OR}=24.287,95 \% \mathrm{CI}$ : 3.114-189.445) and unhealthy dietary patterns $(\mathrm{OR}=10.153,95 \%$ CI: 2.770-37.210) were significantly associated with uncontrolled BP (Table II).

\section{Discussion}

One of the purposes of the study was to assess the rate of uncontrolled BP in patients with predialysis CKD. In this study, $64.8 \%$ of the sample had uncontrolled BP. This finding is consistent with other research. Prior studies have typically found values for uncontrolled BP in the range of $50-75 \%$ among patients with $\mathrm{CKD}$ [9-11]. It was concluded as poor $\mathrm{BP}$ control, and this study's sample was toward the upper end of previously reported findings. However, some previous studies had contradictory findings $[4,18]$. Those studies found that more than $60 \%$ of patients with CKD were able to achieve a BP of $130 / 80 \mathrm{mmHg}$. The variation in the rate of uncontrolled $\mathrm{BP}$ among the studies may be due to the variety of methods used, differences in the study setting and/or sociodemographic variation within-subject populations. In this current study, the subject population was patients with CKD stage $3-5$. One-third of the patients were diagnosed with CKD stage 5 . It is more difficult for patients in the late stage of CKD to control their BP $[16,17]$. Moreover, many of these cases

\begin{tabular}{lclr}
\hline Predictors & OR & $95 \%$ CI & $p$-value \\
\hline Age (years) & & & \\
$\leq 40$ & 1.0 & Reference & \\
$41-60$ & 3.833 & $0.286-51.431$ & 0.310 \\
$>60$ & 3.363 & $0.247-45.792$ & 0.363 \\
Marital status & & & \\
Married & 1.0 & Reference & 0.806 \\
Single & 1.391 & $0.100-19.435$ & \\
Stage of CKD & & & 0.557 \\
Stage 3 & 1.0 & Reference & 0.863 \\
Stage 4 & 1.410 & $0.448-4.440$ & \\
Stage 5 & 1.115 & $0.324-3.840$ & 0.046 \\
Perceived stress & & & 0.001 \\
Low & 1.0 & Reference & \\
Moderate & 4.365 & $1.024-18.609$ & 0.002 \\
High & 10.978 & $2.602-46.312$ & \\
Physical activity & & & $<0.001$ \\
Moderate & 1.0 & Reference & \\
Low & 24.287 & $3.114-189.445$ & \\
Dietary pattern & & & Reference \\
Healthy & 1.0 & $2.770-37.210$ & \\
Unhealthy & 10.153 & &
\end{tabular}

ORs and $95 \%$ CIs for uncontrolled blood pressure
Note(s): Cox \& Snell $R^{2}=0.459$, Nagelkerke $R^{2}=0.632$, predictive correct $=81.8 \%$, Hosmer-Lemeshow test; $\chi^{2}=5.842, \mathrm{df}=8, p$-value $=0.665 ; \mathrm{OR}=$ Odds ratio; $\mathrm{CI}=$ Confidence interval. ${ }^{*} p$-value $<0.05$ 
had diabetes and CVD as comorbidities. These conditions may affect the patient's ability to control their BP. In accordance with the inclusion criteria of the study, the participants had visited the study clinic for more than three months and presumably received appropriate treatment. Yet, some patients reported that they did not know the importance of controlling $\mathrm{BP}$ in delaying $\mathrm{CKD}$ progression. That might be one of the reasons why $\mathrm{BP}$ control was poor in the current study.

Furthermore, the association of lifestyle, response to the environment, physiology, genetic profile and patient's background characteristics with uncontrolled BP was examined. The top two domains in the HDA model (i.e. lifestyle and response to environment) had a significant association with uncontrolled BP in the current study. However, no association between physiology, genetic and patient background characteristics and uncontrolled $\mathrm{BP}$ was found in the sample. These findings are supported by some studies in similar populations $[4,9]$. The related literature revealed that patients in CKD stages 3, 4 and 5 were more likely to have a high awareness of their health issues and receive more aggressive treatment to postpone the progression of the disease [6]. Thus, regardless of a patient's' age, marital status, and stage of $\mathrm{CKD}$, the members of this sample were aware of their condition and were treated without any discrimination by their health care provider. Finally, this finding underlines the necessity of controlling $\mathrm{BP}$ in patients with $\mathrm{CKD}$ in order to minimize the risk of disease progression and complications.

\section{Lifestyle}

According to the Kidney Disease Outcomes Quality Initiative guideline, lifestyle modification plays an important role in managing $\mathrm{BP}$ in the $\mathrm{CKD}$ population [32]. However, lifestyle changes are difficult to achieve in practice. A study about the perception of hypertension management in Asian countries found that $29 \%$ to $51 \%$ of patients made no effort to change their lifestyle, such as taking regular exercise and eating a healthy diet [33]. To some extent, patients believed that taking medication was the most effective way to control their BP. As a result, in the current study, low physical activity and unhealthy dietary patterns were significantly associated with uncontrolled BP in patients with predialysis CKD.

Having a low physical activity was the strongest predictor of uncontrolled BP. It can be seen from the univariate analysis that two-thirds of the sample with low physical activity had uncontrolled BP. In line with previous studies [13, 17], the HDA model explains that low physical activity can be a stressor to the human body, which induces a sympathetic response [12]. Performing regular exercise could decrease the peripheral vascular resistance, neurohormonal and structural responses by lowering sympathetic nerve activity, which plays an important role in the regulation of cardiac performance and peripheral circulation. Thus, the evidence suggests that routine physical activity can improve physical performance and decrease CVD risk.

Likewise, an unhealthy dietary pattern also had a high predictive power for uncontrolled $\mathrm{BP}$ in the present study. That finding is supported by studies conducted in Western and Asian countries [14, 34]. Currently, in Asia (including Indonesia), there is a shift of dietary patterns that mimic western culture [35]. According to the Indonesian Ministry of Health, $93.6 \%$ of the total population was consuming reduced amounts of fruits and vegetables [36]. In this study, almost half of the sample had unhealthy dietary practices. In line with this finding, a study conducted in Indonesia found that approximately one-third of the total population ate fried snacks at a frequency of 4-7 times/week [13]. A study in Bali found that Balinese people had a high sodium intake with a mean of $1,387.14 \mathrm{mg} / \mathrm{day}(\mathrm{SD}=451.98)$ [37]. Further, modifying dietary patterns is recommended by the HDA model [12]. The related literature shows that physiological mechanisms in each individual may contribute to the association between diet and BP [38]. As prior studies have shown, an unhealthy diet
Factors of uncontrolled blood pressure 
JHR 34,6 has become part of the food culture and it is difficult to change. Nevertheless, based on the findings of this study, improving diet would be an important intervention for controlling BP.

\section{Response to the environment}

In this study, perceived stress had a significant association with uncontrolled BP. That finding conforms to results from prior research in a hypertensive population [15]. Even though there are limited data for the CKD population, evidence suggests that CKD places a significant burden on patients due to its chronic condition and its many associated complications [2]. In addition, in this study, $53.3 \%$ of the sample was unemployed, and the majority were in the more productive working-age group. For those possible reasons, as high as $52.8 \%$ of the sample in this study perceived that they have moderate to high levels of stress. According to the HDA model, perceived stress can be a stressor that induces the secretion of hormones by the hypothalamus. Within a certain period, stress will contribute to sympathetic nerve over-activity, which then leads to increased cardiac output. This chain reaction will stimulate the increasing peripheral resistance, which contributes to vascular hypertrophy and thereby linking stress with high BP levels [12]. Thus, based on the findings, stress management is necessary for patients with chronic conditions. Patients need to understand the link between stress and BP regulation. The patient's condition can be improved by healthcare providers, particularly when providing patient counseling. Accordingly, a study conducted in Asian countries recommended that healthcare providers display a more positive attitude, empathy and individual support to patients in order to build trust and improve stress management [33].

In sum, low physical activity, unhealthy dietary patterns (lifestyle factors) and perceived moderate and high stress (response to environmental factors) were significantly associated with uncontrolled BP in this study. Conversely, age, stage of CKD, diabetes mellitus and CVD (physiological factors) along with gender (genetic factors) had no association with uncontrolled BP. Therefore, the findings from this study partially support the HDA model [12].

\section{Implications}

The findings provide evidence for ways in which healthcare providers can improve BP control in their patients. Health care providers need to continue to monitor BP and increase patient education related to the control of BP levels. Promoting lifestyle modification and stress management is necessary since physical activity, dietary patterns and perceived stress were the most important predictive factors of uncontrolled BP. It is recommended that health care providers monitor patients' BP and allow for more individual consultations about lifestyle modification and stress management for patients with chronic conditions. Interventions are needed to address lifestyle modification and stress management, especially in the CKD population.

\section{Limitations}

The study was a cross-sectional study, and convenience sampling was used in collecting the data. Thus, the findings may not provide definitive information about cause and effect relationships, and the results do not necessarily apply to populations with different characteristics. The majority of the sample was male. Duration of and adherence to antihypertensive medications are not known. Therefore, the study needs to be replicated to confirm the findings. Additionally, the sample size in future studies should be increased to enhance the statistical power of tests of significance. 


\section{Conclusions}

$\mathrm{BP}$ control among patients with predialysis $\mathrm{CKD}$ was poor. Physical activity, dietary patterns and stress were statistically significant factors in this study. Healthcare providers are recommended to improve BP control by emphasizing lifestyle modification behaviors and stress management of their patients.

\section{References}

1. Mills KT, Xu Y, Zhang W, Bundy JD, Chen CS, Kelly TN, et al. A systematic analysis of worldwide population-based data on the global burden of chronic kidney disease in 2010. Kidney Int. 2015 Nov; 88(5): 950-7. doi: 10.1038/ki.2015.230. Epub 2015 Jul 29.

2. Stanifer JW, Von Isenburg M, Chertow GM, Anand S. Chronic kidney disease care models in lowand middle-income countries: a systematic review. BMJ Glob Health. 2018 Apr; 3(2): e000728. doi: 10.1136/bmjgh-2018-000728.

3. Riskesdas (Basic Health Research). Basic health research 2013. [Updated: 2013; cited 2018 June 7]. Available from: http://www.depkes.go.id/resources/download/general/Hasil\%20Riskesdas\%202013.

4. Fraser SD, Roderick PJ, McIntyre NJ, Harris S, McIntyre CW, Fluck RJ, et al. Suboptimal blood pressure control in chronic kidney disease stage 3: baseline data from a cohort study in primary care. BMC Fam Pract. 2013 Jun; 14: 88. doi: 10.1186/1471-2296-14-88.

5. Lo C, Zimbudzi E, Teede HJ, Kerr PG, Ranasinha S, Cass A, et al. Patient-reported barriers and outcomes associated with poor glycaemic and blood pressure control in co-morbid diabetes and chronic kidney disease. J Diabetes Complications. 2019 Jan; 33(1): 63-8. doi: 10.1016/j.jdiacomp. 2018.09.020. Epub 2018 Oct 12.

6. Sarafidis PA, Sharpe CC, Wood E, Blacklock R, Rumjon A, Al-Yassin A, et al. Prevalence, patterns of treatment, and control of hypertension in predialysis patients with chronic kidney disease. Nephron Clin Pract. 2012; 120(3): c147-55. doi: 10.1159/000337571.

7. Horowitz B, Miskulin D, Zager P. Epidemiology of hypertension in CKD. Adv Chronic Kidney Dis. 2015 Mar; 22(2): 88-95. doi: 10.1053/j.ackd.2014.09.004.

8. Muntner P, Carey RM, Gidding S, Jones DW, Taler SJ, Wright JT Jr, et al. Potential US population impact of the 2017 ACC/AHA high blood pressure guideline. Circulation. 2018 Jan; 137(2): 109-18. doi: 10.1161/CIRCULATIONAHA.117.032582. Epub 2017 Nov 13.

9. Yan Z, Wang Y, Li S, Wang J, Zhang L, Tan H, et al. Hypertension control in adults with CKD in China: baseline results from the Chinese cohort study of chronic kidney disease (C-stride). Am J Hypertens. 2018 Mar; 31(4): 486-94. doi: 10.1093/ajh/hpx222.

10. Unni S, White K, Goodman M, Ye X, Mavros P, Bash LD, et al. Hypertension control and antihypertensive therapy in patients with chronic kidney disease. Am J Hypertens. 2015 Jun; 28(6): 814-22. doi: 10.1093/ajh/hpu215. Epub 2014 Nov 24.

11. Okaka EI, Ojeh-Oziegbe OE, Unuigbe EI. Factors associated with blood pressure control in predialysis chronic kidney disease patients: short-term experience from a single center in Southern Nigeria. Niger J Clin Pract. 2017 May; 20(5): 537-41. doi: 10.4103/1119-3077.197005.

12. Frazier L. Factors influencing blood pressure: development of a risk model. J Cardiovasc Nurs. 2000 Oct; 15(1): 62-79. doi: 10.1097/00005082-200010000-00007.

13. Peltzer K, Pengpid S. The prevalence and social determinants of hypertension among adults in Indonesia: a cross-sectional population-based national survey. Int J Hypertens. 2018 Aug; 2018: 5610725. doi: 10.1155/2018/5610725.

14. Juraschek SP, Miller ER 3rd, Weaver CM, Appel LJ. Effects of sodium reduction and the DASH diet in relation to baseline blood pressure. J Am Coll Cardiol. 2017 Dec 12; 70(23): 2841-8. doi: 10. 1016/j.jacc.2017.10.011. Epub 2017 Nov 12.

15. Estrada S. The effects of macroaggressions on blood pressure in the Latino population. Sacramento, CA: Alliant International University; 2017.

Factors of uncontrolled blood pressure 
JHR 34,6
16. Lee S, Oh HJ, Lee EK, Lee O, Ha E, Kim SJ, et al. Blood pressure control during chronic kidney disease progression. Am J Hypertens. 2017 Jun; 30(6): 610-6. doi: 10.1093/ajh/hpx017.

17. Zhang W, Shi W, Liu Z, Gu Y, Chen Q, Yuan W, et al. A nationwide cross-sectional survey on prevalence, management and pharmacoepidemiology patterns on hypertension in Chinese patients with chronic kidney disease. Sci Rep. 2016 Dec; 6: 38768. doi: 10.1038/srep38768.

18. Wang LY, Yin DX, Zhang DL, Xu R, Cui WY, Liu WH. Improvement and influencing factors of blood pressure control by nephrologist referral in chronic kidney disease patients in China: a cohort study. Int Urol Nephrol. 2013 Oct; 45(5): 1345-53. doi: 10.1007/s11255-012-0332-8. Epub 2012 Dec 7.

19. Ramezankhani A, Azizi F, Hadaegh F. Associations of marital status with diabetes, hypertension, cardiovascular disease and all-cause mortality: a long term follow-up study. PLoS One. 2019 Apr; 14(4): e0215593. doi: 10.1371/journal.pone.0215593.

20. Alhaddad IA, Hamoui O, Hammoudeh A, Mallat S. Blood pressure control in treated hypertensive Middle Eastern patients: a post hoc analysis based on JNC8 definitions. Vasc Health Risk Manag. 2019 Mar; 15: 35-46. doi: 10.2147/VHRM.S188981.

21. Saeed AA, Al-Hamdan NA, Bahnassy AA, Abdalla AM, Abbas MA, Abuzaid LZ. Prevalence, awareness, treatment, and control of hypertension among Saudi adult population: a national survey. Int J Hypertens. 2011; 2011: 174135. doi: 10.4061/2011/174135. Epub 2011 Sep. 6.

22. KDIGO. KDIGO 2012 Clinical practice guideline for evaluation and management of chronic kidney disease; 2012 [Updated: 2013; cited 2018 July 19]. Available from: http://www.kidneyinternational.org.

23. Whelton PK, Carey RM, Aronow WS, Casey DE, Collins KJ, Dennison Himmelfarb C, et al. 2017 $\mathrm{ACC} / \mathrm{AHA} / \mathrm{AAPA} / \mathrm{ABC} / \mathrm{ACPM} / \mathrm{AGS} / \mathrm{APhA} / \mathrm{ASH} / \mathrm{ASPC} / \mathrm{NMA} / \mathrm{PCNA}$ guideline for the prevention, detection, evaluation, and management of high blood pressure in adults: a report of the American college of cardiology/American heart association task force on clinical practice guidelines. Hypertension. 2018 Jun; 71(6): 1269-324.

24. Cohen S, Kamarck T, Mermelstein R. A global measure of perceived stress. J Health Soc Behav. 1983 Dec; 24(4): 385-96.

25. Lee EH. Review of the psychometric evidence of the perceived stress scale. Asian Nurs. Res. 2012 Dec; 6(4): 121-7. doi: 10.1016/j.anr.2012.08.004. Epub 2012 Sep. 18.

26. Harikedua VT, Tando NM. Relationship between physical activity and eating patterns with the occurrence of central obesity in religious leaders in the city of Manado. Jurnal Gizido. 2012; 4(1): 289-98.

27. Moghaddam MB, Aghdam FB, Jafarabadi MA, Allahverdipour H, Nikookheslat SD, Safarpour S. The Iranian version of international physical activity questionnaire (IPAQ) in Iran: content and construct validity, factor structure, internal consistency and stability. World Appl Sci. 2012; 18(8): 1073-80.

28. IPAQ-Group. IPAQ scoring protocol. 2010; [Updated: cited 2018 June 20]. Available from: https:// sites.google.com/site/theipaq/scoring-protocol.

29. Komrungsee K. Factors associated with blood pressure control in essential hypertensive patients at Suak primary care unit, Nan Province. Bangkok: Mahidol University; 2007.

30. Sauvageot N, Alkerwi A, Albert A, Guillaume M. Use of food frequency questionnaire to assess relationships between dietary habits and cardiovascular risk factors in NESCAV study: validation with biomarkers. Nutr J. 2013 Nov; 12(1): 143. doi: 10.1186/1475-2891-12-143.

31. Sun J, Buys NJ, Hills AP. Dietary pattern and its association with the prevalence of obesity, hypertension and other cardiovascular risk factors among Chinese older adults. Int J Environ Res Public Health. 2014 Apr; 11(4): 3956-71. doi: 10.3390/ijerph110403956.

32. Taler SJ, Agarwal R, Bakris GL, Flynn JT, Nilsson PM, Rahman M, et al. KDOQI US commentary on the 2012 KDIGO clinical practice guideline for management of blood pressure in CKD. Am J Kidney Dis. 2013 Aug; 62(2): 201-13. doi: 10.1053/j.ajkd.2013.03.018. Epub 2013 May 16. 
33. Rahman AR, Wang JG, Kwong GM, Morales DD, Sritara P, Sukmawan R, et al. Asian Cardiovascular Expert Forum Committee. Perception of hypertension management by patients and doctors in Asia: potential to improve blood pressure control. Asia Pac Fam Med. 2015 Feb; 14(1): 2. doi: 10.1186/ s12930-015-0018-3.

34. Herwati H, Sartika W. Blood pressure control of hypertensive patients based on dietary patterns and exercise habits in Padang. Andalas Journal of Public Health. 2011; 8(1): 8-14. 2013.

35. Bishwajit G. Nutrition transition in South Asia: the emergence of non-communicable chronic diseases. Version 2. F1000Res. 2015 Jan; 4: 8. doi: 10.12688/f1000research.5732.2.

36. Ministry of Health Republic of Indonesia. Non-communicable diseases (NCDs) cause most deaths in Indonesia [Updated: 2011 August 18; cited 2018 July 20]. Available from: http://www.depkes.go. id/article/view/1637/penyakit-tidak-menular-ptm-penyebab-kematian-terbanyak-di-indonesia.html.

37. Riska PGA, Aryawangsa N, Agung A, Prabawa I, Manuaba P, Amertha IB, et al. Factors associated with visit-to-visit variability of blood pressure in hypertensive patients at a primary health care service, Tabanan, Bali, Indonesia. Fam Med Community Health. 2018; 6(4): 191-9.

38. Holmes E, Loo RL, Stamler J, Bictash M, Yap IK, Chan Q, et al. Human metabolic phenotype diversity and its association with diet and blood pressure. Nature. 2008 May; 453(7193): 396-400. doi: 10.1038/nature06882.

\section{Corresponding author}

Aurawamon Sriyuktasuth can be contacted at: aurawamon.sri@mahidol.ac.th

For instructions on how to order reprints of this article, please visit our website: 